Pacific Journal of Mathematics

RELATIVE GENERAL POSITION

Vol. 18, No. 3

May 1966 


\section{RELATIVE GENERAL POSITION}

\section{DAVID W. HENDERSON}

In this paper we will say that a piecewise linear map $f: K \rightarrow M$ from a finite complex into an $n$-manifold is a general position (gp) map, if for every pair of simplexes, $A, B$, contained in $K$,

(dimension of the singularities of $f \mid A+B$ )

$$
\leqq(\text { dimension of } A)+(\text { dimension of } B)-n \text {. }
$$

By letting $B=\varnothing$, we see that a gp map into an $n$-manifold is an embedding on each simplex of dimension less than or equal to $n$. Also note that the restriction of a gp map to a subcomplex is again a gp map. It is well known that every map $f$ of a complex into a combinatorial manifold can be homotopically approximated by a gp map, $g$, on some subdivision of the complex. One might suppose that, if $L$ is a subcomplex on which $f$ is already a gp map, then $g \mid L$ could be made equal to $f \mid L$. However, this cannot be done, in general, even if the manifold is a Euclidean space and the complex is a subdivision of a cell. (See the Remark at the end of $\S 3$.)

In $\S 3$ are two general position theorems which fix the map on a subcomplex on which it is already a gp map, but not without some severe restrictions. These theorems are stated in terms of relative general position ( $\mathbf{r g p}$ ) which applied to maps from a pair into a pair. Section 4 considers maps $f:(D$, Bd $D) \rightarrow(M, N)$ of a 2-manifold, $D$, into a 3-manifold, $M$, with 2-submanifold, $N$, with the added restriction that $f($ Bd $D) \cdot f(D-$ Bd $D)=\varnothing$. It is, in general, impossible in this setting to make $f$ into an rgp map while keeping $f \mid \mathrm{Bd} D$ fixed. However, two "relative normal position theorems" are proved which make the singularities "nice" while not considering a particular subdivision.

The proofs are contained in $\S 5$ through 8 .

It should be pointed out that E. C. Zeeman's definition of general position (see [9], p. 59, for general description and [10], Chapter 6, for detailed discussion and proofs) differs from the one used here and avoids most, if not all, of the difficulties encountered in this paper. Thus in a round-about fashion this paper points up several advantages in Zeeman's definition. However, Zeeman's definition may be undesirable for certain purposes. The main difference between the definitions is that Zeeman cannot require that a general position map from a complex $K$ into a manifold to be both in general position on each subcomplex of $K$ and a homeomorphism on each simplex of $K$. 


\section{Definitions.}

2.1. A complex $K$ will be considered to have a given fixed triangulation. $L$ is called a subcomplex of $K$ if $L$ is a complex each of whose simplexes is a simplex of $K . L$ is called a polyhedron in $K$ if $L$ is a subcomplex of some subdivision of $K$.

2.2. If $K$ is a complex and $L$ a subset of $[K]$, then the closed star of $L$ in $K, \operatorname{st}(L, K)$, is the union of all (closed) simplexes of $K$ which intersect $L$. The open star of $L$ in $K$, ost $(L, K)$, is the union of all open simplexes whose closures intersect $L$.

2.3. A mapping, $f$, from a complex $K$ to a complex $L$ is called piecewise linear (pwl) if the graph of $f$ is a polyhedron in the product complex $K \times L$. If $K$ is finite, then there are subdivisions $\alpha, \beta$ such that $f: \alpha K \rightarrow \beta L$ is simplicial.

2.4. A $n$-manifold $M^{n}$ is a separable metric space each of whose points has a closed neighborhood homeomorphic to $I^{n}$, the standard $n$-cell.

The boundary of $M^{n}, \mathrm{Bd} M^{n}$, is the set of points of $M^{n}$ which do not have arbitrarily small neighborhoods homeomorphic to $E^{n}$, $n$-dimensional Euclidean space.

A combinatorial n-manifold is a complex such that the closed star of each vertex has a rectilinear subdivision which is isomorphic to a rectilinear subdivision of an $n$-simplex.

It follows easily from $[1 ; 5]$ that all 2 - and 3 -manifolds may be given combinatorial triangulations, and henceforth in this paper we shall assume that this has been done.

If $N^{p}$ and $M^{n}$ are triangulated manifolds of dimension $p$ and $n$, respectively, then $N^{p}$ is a $p$-submanifold of $M^{n}$ if $N^{p}$ is a subcomplex of $M^{n}$.

2.5. If $f: K \rightarrow L$ is a pwl map of one complex into another, then by the singularities of $f, S(f)$, we shall mean the closure of the set of all points in $L$ which are the images under $f$ of more than one point of $K$. The cardinality of $f^{-1}(x)$ is called the order of $x$.

2.6. Let $L$ be a subcomplex of $K$ and let $N^{p}$ be a polyhedral $p$-submanifold of the $n$-manifold $M^{n}$. A pwl map $f:(K, L) \rightarrow\left(M^{n}, N^{p}\right)$ is said to be a relative general position ( $r g p)$ map if

(a) for every pair of simplexes $(A, B)$ in $K$ such that $B$ is not in $L, \operatorname{dim} S(f \mid A+B) \leqq \operatorname{dim} A+\operatorname{dim} B-n$ 
(b) for every pair of simplexes $(A, B)$ in $L, \operatorname{dim} S(f \mid A+B) \leqq$ $\operatorname{dim} A+\operatorname{dim} B-p$.

2.7. Let $f: J \rightarrow K$ be a pwl map of a 1-manifold into a 2-manifold. We shall call $f$ normal if

(a) $f$ is at most 2-to-1 and $S(f)$ is a finite set of points, and

(b) $f(J)$ crosses itself at each point of $S(f)$.

2.8. Let $C^{3}$ denote the solid cube in $E^{3}$ whose vertices are the eight points in $E^{3}$ which have as each coordinate either 1 or -1 .

2.9. Let $f:(D, \operatorname{Bd} D) \rightarrow\left(M^{3}, N^{2}\right)$ be a pwl map of a 2-manifold $D$ with boundary into a 3 -manifold $M^{3}$ with 2 -submanifold $N^{2}$. We shall call $f$ a relative normal position $(r n p)$ map if

(a ) $f(\mathrm{Bd} D) \cdot f(D-\mathrm{Bd} D)=\varnothing$

(b) $f$ is at most 3 -to-1

(c) $f \mid \mathrm{Bd} D: \mathrm{Bd} D \rightarrow N$ is normal

(d) is a 1-dim polyhedron in $M^{3}$ consisting of (i) double curves (curves along which two sheets of $f(D)$ cross), (ii) triple points (points with arbitrarily small neighborhoods $N$ such that $(N, N \cdot f(D))$ is homeomorphic to $\left(E^{3}\right.$, coordinate planes)), (iii) branch points (points with arbitrarily small closed neighborhoods $N$ such that $(N, N \cdot f(D))$ is homeomorphic to $\left(C^{3}\right.$, cone from the origin over a singular curve on $\left.\mathrm{Bd} C^{3}\right)$ ), and (iv) pinched branch points (points with arbitrarily small closed neighborhoods $V$ such that $(V, V \cdot f(D))$ is homeomorphic to $\left(C^{3}\right.$, cone from origin over two paths on $\left.\left.\mathrm{Bd} C^{3}\right)\right)$.

A pinched branch point is simple if the two paths on $\mathrm{Bd} C^{3}$ are arcs. Note that all pinched branch points must be crossing points of $\mathrm{Bd} D$ on $N^{2}$ because of $(c)$.

2.10. If $f: K \rightarrow M$ and $f: L \rightarrow M$ are maps, then the disjoint sum of $f$ and $g$ is a map $f \oplus g: K \oplus L \rightarrow M$ defined by

$$
(f \oplus g)(x)= \begin{cases}f(x), & \text { if } x \in K \\ g(x), & \text { if } x \in L,\end{cases}
$$

where $K \oplus L$ denotes the abstract disjoint sum of $K$ and $L$.

\section{Relative general position theorems.}

THEOREM I. Let $f:(K, L) \rightarrow(M, N)$ be a pwl map of a pair into a pair, where $L$ is a (ฏp)-dimensional subcomplex of the finite

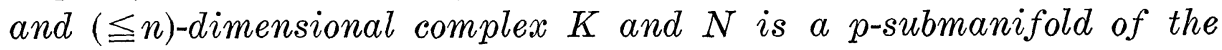
combinatorial n-manifold $M$. Let $\varepsilon$ be a positive number. 
If there exists a subdivision $\alpha$ of $K$ and a pwl map $g: \alpha K \rightarrow M$ such that

$$
g|L=f| L: \alpha L \rightarrow N \text { is a gp map }
$$

(3.2) $g$ takes each closed star of a simplex in $\alpha K$ into the open star of a vertex in $M$,

(3.3) $g$ is a pwl embedding on each simplex of $\alpha K$, and

(3.4) $g$ is obtained from $f$ by a homotopy of $(K, L)$ into $(M, N)$ which leaves $L$ pointwise fixed and moves each point less than $\varepsilon$,

then these exists an rgp map $g^{\prime}:(\alpha K, \alpha L) \rightarrow(M, N)$ that satisfies (3.1)-(3.4).

THeorem II. Let $f:(K, L) \rightarrow(M, N)$ be a pwl map of a pair

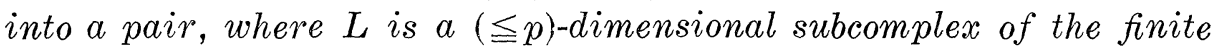

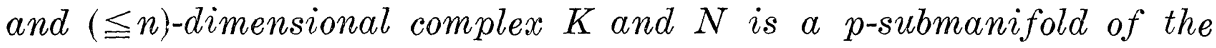
combinatorial n-manifold $M$. Let $\varepsilon$ be a positive number.

If there exists a subdivision $\beta$ of $K$ such that

(3.5) $f \mid L: \beta L \rightarrow N$ is a gp map,

(3.6) $S(f \mid L)$ is a finite set of points, and

(3.7) if $A$ is a simplex in $\beta L$ such that $A \cdot f^{-1}(S(f \mid L))$ is not empty, then $f \mid A$ can be extended to a pwl map $F[A]:$ st $(A, \beta K) \rightarrow M$ which is a homeomorphism on each simplex;

then there exists a subdivision, $\alpha$, of $K$ and an rgp map $g:(\alpha K, \alpha L) \rightarrow(M, N)$ that satisfies (3.1)-(3.4).

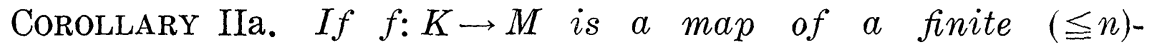
dimensional complex into the combinatorial n-manifold $M$,

then there exists a subdivision, $\alpha$, of $K$ and a pwl gp map $g: \alpha K \rightarrow M$ such that $g$ is arbitrarily close, homotopically, to $f$.

Moreover, if $L$ is a subcomplex of $K$ and $f \mid L$ is a pwl homeomorphism, then we may require that $f|L=f| L$.

(To prove the Corollary, apply the relative simplicial approximation theorem [11] and Theorem II to $f:(K, L) \rightarrow(M, M)$.)

REMARK. Theorem II is false with (3.7) deleted. To see this, let $M=N=E^{4}, K$ be some subdivision of 4 -simplex, and $L=D_{1}+D_{2}$ be the union of two disjoint 2-simplexes on $\mathrm{Bd} K$. Let $D_{1}^{\prime}$ be a polyhedral disk in $E^{4}$ which fails to be locally flat at a point $p$ in the interior of $D_{1}^{\prime}$, and let $D_{2}^{\prime}$ be any polyhedral disk in $E^{4}$ such that 
$D_{1}^{\prime} \cdot D_{2}^{\prime}=p$. (For example, consider $E^{3}$ to be a 3-hyperplane of $E^{4}$ with $p \in E^{4}-E^{3}$, and let $D_{i}^{\prime}$ be the cone from $p$ over a sec $J_{i}$ in $E^{3}$, where $J_{1}$ is knoted in $E^{3}$ and $J_{2}$ links $J_{1}$.) For $i=1,2$, let $f \mid D_{i}$ be a pwl homeomorphism onto $D_{i}^{\prime}$; and then extend to the rest of $K$ in any pwl fashion. $f$ satisfies (3.5) and (3.6) but there is no extension of $f \mid D_{1}+D_{2}$ which is a gp map. This is because if $g: K \rightarrow M=E^{4}$ is an extension of $f \mid D_{1}+D_{2}$ and a homeomorphism on each simplex of some subdivision $\lambda$ of $K$, then, in order that $g \mid \lambda\left(D_{1}+D_{2}\right)$ be a gp map, a neighborhood of $p$ in $D_{1}$ lies on the boundary of the pwl homeomorphic image of a 4 -simplex and thus $D_{1}$ is locally flat at $p$.

QUESTION. Can (3.6) be weakened?

\section{Relative normal position theorems.}

THEOREM III. If $f:(D, J) \rightarrow(M, N)$ is a pwl map of a pair into a pair, where $D$ is a 2-manifold with boundary $J$ and $N$ is a 2-submanifold of the 3-manifold $M$, and, in addition, $f(D-J) \cdot f(J)=\varnothing$, $f \mid J$ is normal, and $\varepsilon$ is a positive number,

then there exists a pwl map $g:(D, J) \rightarrow(M, N)$ such that

(4.1) $g$ is obtained from $f$ by a homotopy of $(D, J)$ into $(M, N)$ which moves each point less than $\varepsilon$ and only moves points at all in an $\varepsilon$-neighborhood of the set of points in $M$ at which $f$ fails to be in rnp (see 2.9),

(4.2) $g$ is a rnp map, and

(4.3) $g|J=f| J$.

Corollary IIIa. If $f: D \rightarrow M$ is a Dehn surface in the 3manifold, $M$, (i.e. $S(f) \cdot f(\operatorname{Bd} D)=\varnothing$ and $f$ is pwl),

then by a "slight adjustment" of $f$ we can get a pwl map $g: D \rightarrow M$ and a neighborhood $N$ of $\mathrm{Bd} D$ in $D$ such that $g|N=f| N$ and $g: D \rightarrow M$ is a normal Dehn surface (i.e. $S(g)$ consists of double curves, branch points, and triple points (see 2.9)).

THEOREM IV. Let $N$ be a 2-submanifold of the 3-manifold $M$ and $h$ be a fixed-point-free pwl homeomorphism of $(M, N)$ onto $(M, N)$ such that hh equals the identity map. Let $\varepsilon$ be a positive number.

If $f:(D, J) \rightarrow(M, N)$ is a pwl map of a 2-manifold $D$ with boundary $J$ into $(M, N)$ such that $(f(D-J)+h f(D-J)) \cdot(f(J)+h f(J))=\varnothing$, and $f \oplus h f \mid J \oplus J$ is normal $[D \oplus D$ is the abstract disjoint union of two copies of $D$ and $f \oplus h f$ is the mapping which is equal to $f$ 
on one copy of $D$ and $h f$ on the other (see 2.10)];

then there exists a pwl map $g:(D, J) \rightarrow(M, N)$ such that

(4.4) $g$ is obtained from $p$ by a homotopy of $(D, J)$ into $(M, N)$ which moves each point less than $\varepsilon$ and only moves points at all in an $\varepsilon$-neighborhood of the set of points in $M$ at which $f \oplus h f$ fails to be in rnp.

(4.5) $g \oplus h g:\left(D_{1}, J_{1}\right) \oplus\left(D_{2}, J_{2}\right) \rightarrow(M, N)$ is a rnp map, and

(4.6) $g|J=f| J$.

Corollary IVa. Let $M$ be a 3-manifold and $h$ a pwl homeomorphism of $M$ onto $M$ such that hh equals the identity map.

If $f: D \rightarrow M$ is an embedding of a surface into $M$ such that $h f(\mathrm{Bd} D) \cdot f(D-\mathrm{Bd} D)=\varnothing$.

then by a "slight adjustment" of $f$ we can get a pwl embedding $g: D \rightarrow M$ and a neighborhood $N$ of $\mathrm{Bd} D$ in $D$ such that $g|N=f| N$ and $g(D) \cdot h g(D)$ is a finite collection of disjoint simple closed curves.

To prove the Corollary apply the Theorem and note that the only singularities possible are double lines.

The Corollaries are used in the various proofs of Dehn's Lemma and the Loop Theorem. (Corollary IIIa is used in [6] and its proof is indicated in [2]. Corollary IVa is used without proof in [7] and [8].) The relative versions in the Theorems are used in the proofs of the author's extensions of Dehn's Lemma and the Loop Theorem, [3] and [4].

It should be noted that if $g: D \rightarrow M$ is a normal Dehn surface, then there are arbitrarily fine subdivisions of $D$ with respect to which $g$ is a gp map. However, there is not necessarily any triangulation with respect to which a given $\mathrm{rnp}$ map is a rgp map, because a nonsimple pinched branch point must be the image of a vertex if the map is a homeomorphism on each simplex.

5. Proof of Theorem I. Let $\alpha L=L_{0} \subset L_{1} \subset L_{2} \subset \cdots \subset L_{k}=\alpha K$, where for $i=0,1,2, \cdots, k-1, L_{i+1}=L_{i}+A_{i}, A_{i}$ is a simplex of $\alpha K$, and $A_{i} \cdot L_{i}=\operatorname{Bd} A_{i}$. (This can be accomplished by adding the simplexes of $\alpha K_{0}$ not in $\alpha L$ in some order of increasing dimension.) Assume inductively that there is a map $g_{m}:(K, L) \rightarrow(M, N)$ such that (let $\left.g_{0}=g\right)$

$(5.1)_{m} g_{m}$ satisfies (3.1)-(3.4) with " $g$ " replaced by " $g_{m}$ ",

$(5.2)_{m} g_{m} \mid L_{m}:\left(L_{m}, \alpha L\right) \rightarrow(M, N)$ is a rgp map, and

$(5.3)_{m} \quad g_{m}\left|K-\operatorname{ost}\left(A_{m-1}, \alpha K\right)=g_{m-1}\right| K-\operatorname{ost}\left(A_{m-1}, \alpha K\right)$ (Note that $L_{m-1} \subset K-\operatorname{ost}\left(A_{m-1}, \alpha K\right)$.) 
Let $P=\operatorname{st}\left(A_{m}, \alpha K\right)$ and $O P=\operatorname{ost}\left(A_{m}, \alpha K\right)$. We shall now alter $g_{m}$ in $P$, keeping it fixed on $P-O P$. Let $Q$ be closed star of a vertex of $M$ such that $g_{m} P \subset$ int $Q$ and let $h$ be a pwl embedding of $Q$ onto the standard $n$-simplex $\Delta$, which we consider as a convex subcomplex of $n$-dimensional Euclidean space, $E^{n}$. Let $\beta$ be a subdivision of $\alpha K$ such that $\beta \alpha\left(g_{m}^{-1}(Q)\right)$ is a subcomplex of $\beta \alpha K$, and

$$
h \circ\left[g_{m} \mid g_{m}^{-1}(Q)\right]: \beta \alpha\left(g_{m}^{-1}(Q)\right) \rightarrow \Delta \subset E^{n}
$$

takes simplexes linearly into simplexes, and such that each simplex of $\beta A$ has at least one vertex in $O P$. Call $h \circ\left[g_{m} \mid g_{m}^{-1}(Q)\right]=H$ and $\beta \alpha\left(g_{m}^{-1}(Q)\right)=R$.

If $C$ is a collection of simplexes in $E^{n}$, let $T(C)$ denote the union of all hyperplanes in $E^{n}$ which contain $n$ vertices of $C$. For a finite collection of simplexes, $C, E^{n}-T(C)$ is open and dense and each of its components is convex.

Let $v_{1}, v_{2}, \cdots, v_{r}$ be the images of those vertices of $\beta A_{m}$ which are not $\beta\left(\mathrm{Bd} A_{m}\right)$. (Remember that $g_{m}$, and therefore $H$, are embeddings on each simplex of $\alpha K$ and therefore are embeddings on each simplex of $\beta \alpha K$.) Let $v_{1}^{*}$ be a point "very close" to $v_{1}$ in $\Delta \cdot\left[E^{n}-T(H(R))\right]$ such that the straight line segment from $v_{1}$ to $v_{1}^{*}$ intersects $T(H(R)$ ) only at $v_{1}$. Define $H_{1}: R \rightarrow \Delta \subset E^{n}$ to be equal to $H$ on $R$-ost $\left(H^{-1} v_{1}, \beta P\right)$ and $H_{1}\left(H^{-1} v_{1}\right)=v_{1}^{*}$ and extend linearly to the rest of st $\left(H^{-1} v_{1}, \beta P\right)$. We leave to the reader the easy verification that $H_{1}$ is an embedding on each simplex of $P$. We now repeat the process with " $v_{2}$ " and " $H_{1}$ " replacing " $v_{1}$ " and " $H$ ", and so forth until we get a map $H^{\prime}=H_{r}$ that is a linear embedding on each simplex of $R$ and a pwl embedding on each simplex of $P$, and such that, for each $i, H^{\prime}\left(H^{-1} v_{i}\right)=v_{i}^{*}$ belongs to $\left[E^{n}-T\left(H(R-O P)+v_{1}^{*}+\cdots+v_{i-1}^{*}\right)\right] \cdot \Delta$. (Note that $H\left|R-O P=H_{1}\right| R-O P=H_{2}\left|R-O P=\cdots=H^{\prime}\right| R-O P$.) Define $g_{m+1}\left|\alpha K-O P=g_{m}\right| \alpha K-O P$ and $g_{m+1}\left|P=h^{-1} H\right| P$.

We must now show that $(5.1)_{m+1}-(5.3)_{m+1}$ are satisfied. $(5.1)_{m+1}$ is satisfied if we make $v_{i}$ close enough to $v_{i}^{*}$. (5.3) $)_{m+1}$ is inherent in the construction. If $(5.2)_{m+1}$ is not satisfied then there is a $b$-dimensional simplex $B$ in $\alpha K$ such that (let $a=$ dimension of $A_{m}$ )

$$
\operatorname{dimension}\left(S\left(g_{m+1} \mid A_{m}+B\right)\right)>b+a-n \text {. }
$$

Then there would be a $(\leqq b)$-dimensional simplex $B^{\prime}$ in $\beta B \cdot R$ and a ( $\leqq a)$-dimensional simplex $A^{\prime}$ in $\beta A_{m}$ such that

$$
\text { dimension }\left(\left(g_{m+1} A^{\prime} \cdot g_{m+1} B^{\prime}\right)-g_{m+1}\left(A^{\prime} \cdot B^{\prime}\right)\right)>b+a-n \text {. }
$$

However, if $v_{t}^{*}$ is the one member of $\left\{v_{1}^{*}, \cdots, v_{r}^{*}\right\} \cdot g_{m+1} A^{\prime}$ with highest subscript, then it is a straightforward exercise in linear algebra to show that $v_{t}^{*}$ belongs to 


$$
T\left[g_{m+1}\left(A^{\prime}+B^{\prime}\right)-\operatorname{ost}\left(v_{t}^{*}, g_{m+1} A^{\prime}\right)\right] \subset T\left(H(R-O P)+v_{1}^{*}+\cdots+v_{t-1}^{*}\right)
$$

which contradicts our restrictions on the choice of $v_{t}^{*}$.

Thus we may conclude that there exists a map $g^{\prime}=g_{k}$ that satisfies $(5.1)_{k}-(5.3)_{k}$. This is the map required in Theorem I.

\section{Proof of Theorem II. First, we prove a necessary}

LEMma. Let $C$ be pwl homeomorphic (under $h$ ) to the standard

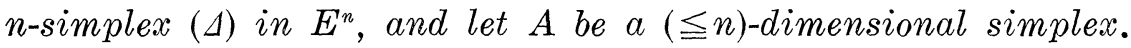

If $f: \alpha A \rightarrow C$ is a pwl map which is a homeomorphism on each simplex of $\alpha(\mathrm{Bd} A)$, for some subdivision $\alpha$ of $A$,

then $f$ is arbitrarily close, homotopically, to a pwl map $g: \pi A \rightarrow C$ such that $g|\mathrm{Bd} A=f| \mathrm{Bd} A$ and $g$ is a homeomorphism on each simplex of $\pi A$, where $\pi$ is some subdivision of $A$ and refines $\alpha$.

Proof. Let $\pi$ be a subdivision of $A$ such that $\pi A$ is a subdivision of $\alpha A$ and $h f: \pi A \rightarrow C \rightarrow \Delta \subset E^{n}$ is linear on each simplex of $\pi A$. Further, assume that, for each simplex $B$ in $\pi A, B \cdot \mathrm{Bd} A$ is a single face (possibly empty) of $B$. Let $v_{1}, \cdots, v_{r}$ be the vertices of $\pi A$ not in $\pi(\operatorname{Bd} A)$. Let $v_{1}^{\prime}, \cdots, v_{r}^{\prime}$ be a collection of points in $\Delta$ such that, for each $i, v_{i}^{\prime}$ is "close" to $h f\left(v_{i}\right)$ and $v_{i}^{\prime}$ does not lie on any linear subspace of $E^{n}$ that is determined by some collection of $k(\leqq n)$ points from $\left\{v_{1}^{\prime}, \cdots, v_{i-1}^{\prime}, v_{i+1}^{\prime}, \cdots, v_{r}^{\prime}\right\}+h f$ (the vertices of $\left.\pi(\mathrm{Bd} A)\right)$. Define $g|\mathrm{Bd} A=f| \mathrm{Bd} A, g\left(v_{i}\right)=v_{i}^{\prime}$ for each $i$, and then extend linearly to the rest of $A$. By making $v_{i}^{\prime}$ close enough to $h f\left(v_{i}\right)$, for each $i$, we can make $g$ as close, homotopically, to $f$ as we please. It is an easy exercise to show that $g$ is a homeomorphism on each simplex of $\pi A$.

We now return to the proof of Theorem II. By the carrier of a point, $p$, in $\beta K$ we shall mean the unique simplex of $\beta K$ which contains $p$ in its interior. Note that any subdivision $\mu$ of $\beta K$ satisfies (3.5)-(3.7) if, for every point $p$ of $f^{-1}(S(f \mid L))$, the carrier of $p$ in $\mu \beta K$ has the same dimension as the carrier of $p$ in $\beta K$. Therefore we may assume without loss of generality that $\beta$ is so fine that, for every simplex $A$ of $\beta K, f(s t(A, \beta K))$ is contained in some open star of a vertex of $M$. Let $A_{1}, \cdots, A_{s}$ be the collection of carriers of points of $f^{-1}(S(f \mid L))$. Since $A_{i}$ is compact and there is a pwl homeomorphism, keeping $A_{i}$ fixed, of $\operatorname{st}\left(A_{i}, \beta K\right)$ into any neighborhood of $A_{i}$ in $\operatorname{st}\left(A_{i}, \beta K\right)$, we may assume that, for each $i, F\left[A_{i}\right]\left(\operatorname{st}\left(A_{i}, \beta K\right)\right)$ is contained in the same open star of a vertex as $f\left(\operatorname{st}\left(A_{i}, \beta K\right)\right)$. We also suppose that $f\left[A_{i}\right]=f \mid \operatorname{st}\left(A_{i}, \beta K\right)$, if the latter is a homeomorphism on each simplex. We can now easily obtain a pwl map $f^{*}:(\beta K, \beta L) \rightarrow(M, N)$ such that $f^{*}|L=f| L$ and $f^{*} \mid \operatorname{st}\left(A_{i}, \beta K\right)=F\left[A_{i}\right]$ and such that (3.4) is satisfied with " $g$ " replaced by " $f *$ ". Let $B_{1}, \cdots, B_{t}$ be the simplexes of $\beta K$ 
not in $\beta L+\sum\left[\operatorname{st}\left(A_{i}, \beta K\right)\right]$ and suppose that they are arranged in some order of increasing dimension. Let

$$
L_{j}=\beta L+\sum_{i=1}^{i=s}\left[\operatorname{st}\left(A_{i}, \beta K\right)\right]+\sum_{k=1}^{k=j}\left[B_{k}\right],
$$

for $j=0,1, \cdots, t$. Note that $\mathrm{Bd} B_{j+1} \subset L_{j}$. We assume inductively that we have a subdivision $\mu_{j}$ of $K$ and a pwl map $f_{j}:\left(\mu_{j} K, \mu_{j} L\right) \rightarrow$ $(M, N)$ such that

$(6.1)_{j} f_{j}$ is a homeomorphism on each simplex of $\mu_{j} L_{j}$,

$(6.2)_{j} \quad f_{j}\left|L_{0}=f^{*}\right| L_{0}$,

$(6.3)_{j} \quad \mu_{j} K$ is a subdivision of $\beta K$,

$(6.4)_{j}$ each $A_{i}$ is a simplex of $\mu_{j} K$,

$(6.5)_{j} f_{j}$ maps the star of each simplex of $\beta K$ into the open star of a vertex of $M$, and

$(6.6)_{j} \quad f_{j}$ is obtained from $f$ by a homotopy of $(K, L)$ into $(M, N)$ which leaves $L$ pointwise fixed and moves each point less than $\varepsilon$.

Note that $f^{*}=f_{0}$ satisfies $(6.1)_{0}-(6.6)_{0}$. We will now use the Lemma and $\mu_{j}$ and $f_{j}$ to construct $\mu_{j+1}$ and $f_{j+1}$. Let $C$ be the open star of some vertex of $M$ such that $f_{j}\left(\operatorname{st}\left(B_{j+1}, \beta K\right)\right) \subset C$. Thus we can apply the Lemma and obtain a pwl map $g: \pi B_{j+1} \rightarrow C$ arbitrarily close, homotopically, to $f_{j} \mid B_{j+1}$ such that $g\left|\mathrm{Bd} B_{j+1}=f_{j}\right| B_{j+1}, g$ is a homeomorphism on each simplex of $\pi B_{j+1}$, and $\pi\left(\mathrm{Bd} B_{j+1}\right)$ is a subdivision of $\mu_{j}\left(\mathrm{Bd} B_{j+1}\right)$. Then define $\mu_{j+1}\left(B_{j+1}\right)=\pi\left(B_{j+1}\right)$ and extend to a subdivision of $\mu_{j} K$. Since $\operatorname{st}\left(A_{i}, \beta K\right)$ is contained in $L_{0}$ and therefore in $L_{j}$, we may do the extending in such a way that $(6.4)_{j+1}$ is satisfied. We define $f_{j+1} \mid B_{j+1}=g$ and

$$
f_{j+1}\left|K-\operatorname{ost}\left(B_{j+1}, \beta K\right)=f_{j}\right| K-\operatorname{ost}\left(B_{j+1}, \beta K\right)
$$

and then extend (in $C$ ) to the rest of $\operatorname{st}\left(B_{j+1}, \beta K\right)$. It is clear that if $g$ is close enough to $f_{j} \mid B_{j+1}$ then $(6.5)_{j+1}$ and $(6.6)_{j+1}$ will be satisfied. $(6.1)_{j+1}$ is satisfied because $\mu_{j+1} K$ is a subdivision of $\mu_{j} K$.

Thus by induction we may assume that there is a pwl map $f_{t}:\left(\mu_{t} K, \mu_{t} L\right) \rightarrow(M, N)$ that satisfies $(6.1)_{t}-(6.6)_{t}$. We now show that $f_{t}$ and $\mu_{t}$ satisfy (3.1)-(3.4) with " $g$ " replaced by " $f_{t}$ " and " $\alpha$ " replaced by " $\mu_{t}$ ". (3.1) follows from $(6.2)_{t}-(6.4)_{t},(3.2)$ follows from $(6.5)_{t},(3.3)$ follows from $(6.1)_{t}$, and (3.4) follows from $(6.6)_{t}$.

Thus Theorem II now follows from Theorem I.

7. Proof of Theorem III. We first obtain a subdivision $\lambda$ of $D$ and a pwl map $g^{\prime}: \lambda D \rightarrow M$ that satisfies (4.1) and (4.2) with " $g$ " 
replaced by " $g$ '", and so that $g^{\prime}$ is a homeomorphism on each simplex of $\lambda D$. The proof that such a $\lambda$ and $g^{\prime}$ exist follows closely the arguments in $\S 6$, with the arguments dealing with the $A_{i}^{\prime}$ s omitted; and therefore we will not give the details here. Suffice it to say that one should find a subdivision $\beta$ of $M$ so that $\varepsilon$ is larger than the diameter of each vertex star of $\beta M$ and then require that the image under $f$ and $g^{\prime}$ of each vertex star of $\lambda D$ must be contained in the open star of a vertex of $M$.

Let $\alpha$ and $\delta$ be subdivision of $M$ and $D$, respectively, so that $g^{\prime}$ is simplicial from $\delta D$ to $\alpha M$. Then $g^{\prime}(D)$ is a subcomplex of $\alpha M$ and $S\left(g^{\prime}\right)$ is a subcomplex of $g^{\prime}(D)$. Let $\alpha^{2}$ denote the second barycentric subdivision of $\alpha$. Note that $S\left(g^{\prime}\right) \cdot g^{\prime}(J)$ is just the crossing points of $g^{\prime} \mid J$.

If $\omega$ is a 2-simplex of $S\left(g^{\prime}\right)$, then $g^{\prime-1}(\omega)$ is the union of two or more 2-simplexes of $D$ and $\operatorname{st}\left(\omega, \alpha^{2} M\right)$ is a 3-cell of which $\omega$ is a spanning disk. Being careful not to move things too far we can adjust the interiors of the images of each 2-simplex of $g^{\prime-1}(\omega)$ so that they still lie in $\operatorname{st}\left(\omega, \alpha^{2} M\right)$ but are disjoint except for their boundaries. By applying this procedure to each 2-simplex in $S\left(g^{\prime}\right)$ we can get a pwl map $g^{\prime \prime}: D \rightarrow M$ that satisfies (4.1) and (4.3) and $S\left(g^{\prime \prime}\right)$ is a 1subcomplex of $\alpha M$.

If $\tau$ is a 1-simplex of $S\left(g^{\prime \prime}\right)$, then $\operatorname{st}\left(\tau, \alpha^{2} M\right)$ is a 3-cell of which $\tau$ is a spanning arc and $g^{\prime \prime-1}\left(\operatorname{st}\left(\tau, \alpha^{2} M\right)\right)$ is the union of two or more subdisks of $D$, the image of each being a spanning disk of $\operatorname{st}\left(\tau, \alpha^{2} M\right)$. We may then alter these spanning disks slightly in the interior of st $\left(\tau, \alpha^{2} M\right)$ so that the only singularities in the interior of $\operatorname{st}\left(\tau, \alpha^{2} M\right)$ are double lines whose endpoints are the endpoints of $\tau$. In this fashion we can obtain a pwl map $g^{\prime \prime \prime}$ that satisfies (4.1) and (4.3) and $g^{\prime \prime \prime}$ fails to be a rnp map only at the vertices of $S\left(g^{\prime \prime}\right)$. Analogously, we adjust slightly the images in $\operatorname{st}\left(v, \alpha^{2} M\right)$ for each vertex, $v$, of $S\left(g^{\prime \prime}\right)-g^{\prime \prime}(J)$ so that the only singularities in $\operatorname{st}\left(v, \alpha^{2} M\right)$ are triple points, branch points, and the ends of double lines. The pwl map then obtained is the desired map $g$. The vertices of $S\left(g^{\prime \prime}\right) \cdot g^{\prime \prime}(J)$ are just the crossing points of $g^{\prime \prime \prime}\left|J=g^{\prime \prime}\right| J$ and thus the pinched branch points of $g^{\prime \prime \prime} \mid D$.

8. Proof of Theorem IV. First we wish to pick subdivisions of $D$ and $M$ so that both $f$ and $h$ are simplicial with respect to these subdivisions. Let $\nu$ and $\beta$ be subdivisions so that $f: \nu D \rightarrow \beta M$ is simplicial. Since $h$ is pwl, there exist refinements $\beta_{1}$ and $\beta_{2}$ of $\beta$ such that $h: \beta_{1} M \rightarrow \beta_{2} M$ is simplicial. Let $\left(\beta_{1} \cdot \beta_{2}\right) M$ denote the convex linear cell complex composed of all cells of the form $\tau_{1} \cdot \tau_{2}$ where $\tau_{i}$ is a simplex of $\beta_{i} M$. (See [10], Chapter 1, page 5, for a description of 
convex linear cell complex.) Now the image under $h$ of a cell of $\left(\beta_{1} \cdot \beta_{2}\right) M$ is again a cell of $\left(\beta_{1} \cdot \beta_{2}\right) M$. Then, order the cells of $\left(\beta_{1} \cdot \beta_{2}\right) M$ in some order of increasing dimension, $\mu_{1}, \mu_{2}, \cdots, \mu_{p}$, so that for each $t, \mu_{2 t}=h\left(\mu_{2 t-1}\right)$. We now subdivide, one at a time in order, each cell into a simplex, while leaving the subdivision fixed on the boundary of the cell; and in doing this we let $h\left(\mu_{2 t-1}\right)$ determine the subdivision on $\mu_{2 t}$, for each $t$. (See [10], Chapter 1, Lemma 1.) In this way we obtain a subdivision $\alpha$ of $M$ that refines $\beta$, and such that $h: \alpha M \rightarrow \alpha M$ is simplicial. By Lemma 5 of Chapter 1 of [10], there is a subdivision $\delta$ of $D$ such that $f: \delta D \rightarrow \alpha M$ is simplicial.

Theorem IV can now be proved using essentially the same arguments used in the proof of Theorem III, with the exception that all subdivisions of $M$ should be such that $h$ is simplicial with respect to them and in altering the maps the changes made in the star of a simplex should "agree" with the changes of the maps in the image of that star under $h$.

\section{REFERENCES}

1. R. H. Bing, Locally tame sets are tame, Ann. of Math., 59 (1954), 145-158.

2. M. Dehn, Über die topologie des dreidimensionalen raumes, Math. Ann. 69 (1910), 137-168.

3. D. W. Henderson, Extensions of Dehn's lemma and the loop theorem, Trans. Amer. Math. Soc. 120 (1965), 448-469.

4. - Self-unlinked simple closed curves, Trans. Amer. Math. Soc. 120 (1965), $470-480$.

5. E. E. Moise, Affine structures in 3-manifolds, $V$. The triangulation theorem and hauptvermutung, Ann. of Math. 56 (1952), 96-114.

6. C. D. Papakyriakopoulos, Dehn's lemma and the asphericity of knots, Ann. of Math. 66 (1957), 1-26.

7. A. Shapiro and J. H. C. Whitehead, A proof and extension of Dehn's lemma, Bull. Amer. Math. Soc. 64 (1958), 174-178.

8. J. Stallings, On the loop theorem, Ann. of Math. 72 (1960), 12-19.

9. E. C. Zeeman, Polyhedral Manifolds, Topology of 3-manifolds, ed. by M. K. Fort, 1961.

10. - Seminar on Combinatorial Topology, Institut des Hauptes Etudes Scientifiques, 1963, (mimeographed).

11. - Relative simplicial approximation, Proc. Camb. Phil. Soc. 60 (1964), $39-43$.

Received January 30, 1965. This research was supported by a grant from The Institute for Advanced Study.

The Institute For Advanced Study

PRINCETON, N.J. 



\section{PACIFIC JOURNAL OF MATHEMATICS}

\section{EDITORS}

\author{
H. SAMELSON, \\ Stanford University \\ Stanford, California \\ R. M. BLUMENTHAL \\ University of Washington \\ Seattle, Washington 98105
}

\author{
*J. DugundJI \\ University of Southern California \\ Los Angeles, California 90007 \\ RICHARD ARENS \\ University of California \\ Los Angeles, California 90024
}

\section{ASSOCIATE EDITORS}
E. F. BECKENBACH
B. H. NeUmanN
F. WOLF
K. YOSIDA

\section{SUPPORTING INSTITUTIONS}

\author{
UNIVERSITY OF BRITISH COLUMBIA \\ CALIFORNIA INSTITUTE OF TECHNOLOGY \\ UNIVERSITY OF CALIFORNIA \\ MONTANA STATE UNIVERSITY \\ UNIVERSITY OF NEVADA \\ NEW MEXICO STATE UNIVERSITY \\ OREGON STATE UNIVERSITY \\ UNIVERSITY OF OREGON \\ OSAKA UNIVERSITY \\ UNIVERSITY OF SOUTHERN CALIFORNIA
}

\author{
STANFORD UNIVERSITY \\ UNIVERSITY OF TOKYO \\ UNIVERSITY OF UTAH \\ WASHINGTON STATE UNIVERSITY \\ UNIVERSITY OF WASHINGTON \\ * * * \\ AMERICAN MATHEMATICAL SOCIETY \\ CHEVRON RESEARCH CORPORATION \\ TRW SYSTEMS \\ NAVAL ORDNANCE TEST STATION
}

Mathematical papers intended for publication in the Pacific Journal of Mathematics should be typewritten (double spaced). The first paragraph or two must be capable of being used separately as a synopsis of the entire paper. It should not contain references to the bibliography. Manuscripts may be sent to any one of the four editors. All other communications to the editors should be addressed to the managing editor, Richard Arens at the University of California, Los Angeles, California 90024 .

50 reprints per author of each article are furnished free of charge; additional copies may be obtained at cost in multiples of 50 .

The Pacific Journal of Mathematics is published monthly. Effective with Volume 16 the price per volume (3 numbers) is $\$ 8.00$; single issues, $\$ 3.00$. Special price for current issues to individual faculty members of supporting institutions and to individual members of the American Mathematical Society: $\$ 4.00$ per volume; single issues $\$ 1.50$. Back numbers are available.

Subscriptions, orders for back numbers, and changes of address should be sent to Pacific Journal of Mathematics, 103 Highland Boulevard, Berkeley 8, California.

Printed at Kokusai Bunken Insatsusha (International Academic Printing Co., Ltd.), No. 6, 2-chome, Fujimi-cho, Chiyoda-ku, Tokyo, Japan.

PUBLISHED BY PACIFIC JOURNAL OF MATHEMATICS, A NON-PROFIT CORPORATION

The Supporting Institutions listed above contribute to the cost of publication of this Journal, but they are not owners or publishers and have no responsibility for its content or policies.

* Paul A. White, Acting Editor until J. Dugundji returns. 


\section{Pacific Journal of Mathematics}

\section{Vol. 18, No. 3 \\ May, 1966}

William George Bade and Philip C. Curtis, Jr., Embedding theorems for commutative Banach algebras .......................... 391

Wilfred Eaton Barnes, On the $\Gamma$-rings of Nobusawa ................. 411

J. D. Brooks, Second order dissipative operators ................ 423

Selwyn Ross Caradus, Operators with finite ascent and descent ........ 437

Earl A. Coddington and Anton Zettl, Hermitian and anti-hermitian properties of Green's matrices .......................... 451

Robert Arnold Di Paola, On sets represented by the same formula in distinct consistent axiomatizable Rosser theories ................... 455

Mary Rodriguez Embry, Conditions implying normality in Hilbert space ...........................................

Garth Ian Gaudry, Quasimeasures and operators commuting with convolution ................................... 461

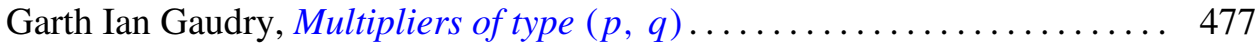

Ernest Lyle Griffin, Jr., Everywhere defined linear transformations affiliated with rings of operators .................................. 489

Philip Hartman, On the bounded slope condition ................ 495

David Wilson Henderson, Relative general position ................ 513

William Branham Jones, Duality and types of completeness in locally

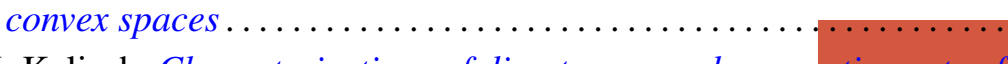

G. K. Kalisch, Characterizations of direct sums and commuting sets of Volterra operators....

Ottmar Loos, Über eine Beziehung zwischen Malcev-Algebren und Lietripelsystemen

Ronson Joseph Warne, A class of bisimple inverse semigroups . . . 